PHYSICAL REVIEW D 93, 089905(E) (2016)

\title{
Erratum: Damped and zero-damped quasinormal modes of charged, nearly extremal black holes \\ [Phys. Rev. D 93, 044033 (2016)]
}

\author{
Aaron Zimmerman and Zachary Mark \\ (Received 25 March 2016; published 8 April 2016)
}

DOI: 10.1103/PhysRevD.93.089905

In the original paper, footnotes 2 and 3 incorrectly describe the claims of Refs. [1,2], respectively. Those papers in fact state that their analytic formulas deal only with the scalar and neutrino modes of Kerr-Newman black holes. The error was due to our misunderstanding of the papers' claims, and the footnotes should be removed entirely. These issues do not impact the other results or conclusions of our paper.

[1] S. Hod, Phys. Lett. B 666, 483 (2008).

[2] S. Hod, Eur. Phys. J. C 75, 272 (2015). 